\title{
PRÁTICAS DE IDENTIFICAÇÃO DO PACIENTE EM UNIDADE DE TERAPIA INTENSIVA PEDIÁTRICA
}

\author{
Suelen Cristina Zandonadi Bernal ${ }^{1}$, Daiane Cortez Raimondi², João Lucas Campos de Oliveira ${ }^{3}$, \\ Kelly Cristina Inoe ${ }^{4}$, Laura Misue Matsuda ${ }^{5}$
}

\begin{abstract}
Objetivo: investigar práticas de identificação do paciente em unidade de terapia intensiva pediátrica. Método: estudo transversal realizado em três hospitais públicos do Sul do Brasil. A coleta de dados (itens identificadores) foi realizada em 2015, por meio de observação não participante e consulta em prontuário. Os dados foram analisados por estatística descritiva. Resultados: dos 96 pacientes observados, 94 (98\%) tinham identificação próxima ao leito, por placa. Não houve nenhuma identificação por meio de pulseira. Nas placas identificadoras, constavam o nome da criança (95\%), a idade (31\%), e o nome do responsável (78\%). Quando identificados pelo nome $(n=89), 62(70 \%)$ eram completos. Na prescrição médica e evolução de enfermagem, houve deficiências nos registros do nome completo, da data de nascimento e do nome dos pais. Conclusão: Apesar da vasta gama de recursos, há deficiência na sistematização da identificação do paciente, sendo uma implicação ao cuidado (in)seguro.
\end{abstract}

DESCRITORES: Sistemas de identificação de pacientes; Segurança do paciente; Gestão de riscos; Enfermagem pediátrica; Unidades de terapia intensiva.

\section{PATIENT IDENTIFICATION PRACTICES IN A PEDIATRIC INTENSIVE CARE UNIT}

Objective: to investigate patient identification practices in a pediatric intensive care unit. Method: a cross-sectional study was carried out in three public hospitals in the South of Brazil. Data collection (identifying items) was performed in 2015, through non-participant observation and consultation of the medical records. The data were analyzed using descriptive statistics. Results: of the 96 patients observed, 94 (98\%) had an identification nameplate next to the bed. No identification wristbands were used. The identification nameplates included the child's name (95\%), age (31\%) and the name of the person responsible (78\%). Of those identified by name $(n=89), 62(70 \%)$ were complete. In the medical prescription and nursing record there were deficiencies in the registration of the full name, date of birth and names of the parents. Conclusion: despite the wide range of resources, there was a deficiency in the systematization of patient identification, affecting (un)safe care.

KEYWORDS: Patient identification systems; Patient safety; Risk management; Pediatric nursing; Intensive care units.

\section{PRÁCTICAS DE IDENTIFICACIÓN DEL PACIENTE EN UNIDAD DE TERAPIA INTENSIVA PEDIÁTRICA}

Objetivo: investigar prácticas de identificación del paciente en unidad de terapia intensiva pediátrica. Método: estudio transversal que se realizó en tres hospitales públicos del Sur de Brasil. Se recogieron los datos (identificadores) en 2015, por medio de observación no participante y consulta en prontuario. Se analizaron esos datos por estadística descriptiva. Resultados: de los 96 pacientes observados, 94 (98\%) tenían identificación cerca del lecho, por placa. No hubo identificación por medio de brazalete. En las placas identificadoras, había el nombre del niño (95\%), la edad (31\%) y el nombre del responsable (78\%). Cuando estaban identificados por el nombre ( $n=89), 62(70 \%)$ estos eran completos. En la prescripción médica y evolución de enfermería, hubo deficiencias en los registros de nombre completo, fecha de nacimiento y nombre de los padres. Conclusión: A pesar de la gran cantidad de recursos, hay deficiencia en la sistematización de la identificación del paciente, siendo esa una implicación del cuidado (in) seguro.

DESCRIPTORES: Sistemas de identificación de pacientes; Seguridad del paciente; Gestión de riesgos; Enfermería pediátrica; Unidades de terapia intensiva.

1Enfermeira. Mestre em Enfermagem. Universidade Estadual de Maringá (UEM). Maringá, PR, Brasil.

2 Enfermeira. Mestre em Enfermagem. Docente da Universidade Paranaense(UNIPAR). Umuarama, PR, Brasil.

3 Enfermeiro. Doutor em Enfermagem. Docente da Universidade Estadual do Oeste do Paraná (UNIOESTE). Cascavel-PR, Brasil.

4 Enfermeira. Doutora em Enfermagem. Universidade Estadual de Maringá (UEM). Maringá-PR, Brasil.

5 Enfermeira. Doutora em Enfermagem. Docente do Programa de Pós-Graduação em Enfermagem. Universidade Estadual de Maringá (UEM). Maringá, PR, Brasil. 


\section{INTRODUÇÃO}

A complexidade do setor saúde, a dinâmica da evolução social, econômica, epidemiológica e a necessidade de concatenar os objetivos organizacionais com a satisfação dos clientes são fatores que devem posicionar a qualidade como preocupação legítima dos gestores. ${ }^{(1)}$

No sentido de alavancar a qualidade dos serviços de saúde, algumas dimensões do cuidado alicerçam a sua concepção, tais como: eficácia, eficiência, acessibilidade, aceitabilidade, equidade e, mais recentemente, a segurança. ${ }^{(2)}$ Assim, na saúde, a segurança do paciente é compreendida como a redução, a um mínimo aceitável, do risco associado à assistência. ${ }^{(3)}$

A fim de maximizar o cuidado seguro, a Organização Mundial de Saúde (OMS) estabeleceu na primeira década dos anos 2000 seis metas para a segurança do paciente, (2) as quais são consideradas como desafio real a ser enfrentado no contexto da saúde, inclusive no Brasil. ${ }^{(3,4)}$

Proposta como a primeira meta para o atendimento seguro, ${ }^{(5)}$ a identificação do paciente é considerada fator prioritário nas instituições de saúde, porque a sua principal finalidade é garantir que o cuidado seja executado no paciente certo, com intuito de evitar a ocorrência de eventos adversos relacionados à assistência. ${ }^{(6,7)}$ Logo, a identificação do paciente é de extrema importância, pois, no caso de erro inicial na identificação, o fim da assistência tenderá à ocorrência de algum evento adverso, ou seja, uma consequência danosa, de magnitude diversa, com impacto real à saúde do paciente. ${ }^{(7)}$

Sabe-se que alguns fatores podem elevar as chances de erro no processo de identificação do paciente, ao exemplo da mudança de leito, estado de consciência do paciente, patologias que causem afasia, falta de acompanhante, entre outros. ${ }^{(7)}$ Com isso, evidencia-se ainda mais a necessidade de supervisão e controle do processo de identificação do paciente para que essa medida, de fato, favoreça o atendimento seguro. ${ }^{(8)}$

No Brasil, a identificação do paciente tem sido recomendada da seguinte forma: uso de pelo menos dois identificadores em pulseira branca padronizada pela instituição, colocada em um membro do paciente para que seja conferida antes do cuidado $^{(7)}$. Além disso, outras iniciativas de segurança compreendem o protocolo de identificação do paciente, bem como o processo de identificação deve ser cumprido em todos os ambientes de prestação de cuidado de saúde, independentemente do tipo de assistência realizada. ${ }^{(7)}$

Reconhece-se que o paciente pediátrico tem maior chance de sofrer algum dano à saúde durante a sua hospitalização por possuir particularidades morfológicas, desenvolvimento cognitivo, social e emocional diferenciados do adulto. Nesse sentido, pode apresentar mudanças rápidas e alterações no estado clínico, com necessidade de procedimentos diagnósticos e terapêuticos complexos que comumente se vinculam à assistência intensiva e/ou semicrítica. ${ }^{(9)}$

Estudo $^{(10)}$ realizado na Espanha, com finalidade de identificar riscos potenciais aos pacientes pediátricos, identificou 49 oportunidades de falhas, 60 consequências e 252 causas. A alta do paciente foi identificada como a principal falha, enquanto as queixas dos pais quanto à longa permanência de internação, atraso no diagnóstico ou tratamento e tratamento desnecessário foram as consequências mais comuns. Quanto às principais causas dos riscos, constatou-se não incluir a família no processo assistencial, mudança de turno e identificação incorreta do paciente. ${ }^{(10)}$

Alude-se que investigar acerca das práticas de identificação utilizadas em paciente pediátrico internado em regime de terapia intensiva é social e cientificamente importante, em virtude de que os seus resultados podem subsidiar decisões que confluam ao atendimento seguro dessa peculiar clientela. Com base nessas premissas, questiona-se: Como se apresentam as práticas de identificação do paciente pediátrico crítico? E, para responder a esta questão, propõe-se a realização deste estudo que tem como objetivo investigar práticas de identificação do paciente em Unidades de Terapia Intensiva Pediátrica (UTI-P). 
Estudo descritivo, transversal, e de abordagem quantitativa. Foi realizado nas UTI-P de três hospitais universitários gerais públicos de médio e grande porte, situados na região Sul do Brasil. Destes, dois eram de administração estadual e um federal.

A coleta de dados foi realizada nas UTI-P dos referidos hospitais, durante 10 dias consecutivos e aleatórios, no período de fevereiro a maio de 2015, por meio da técnica de Observação não Participante ou Observação Sistemática, a qual se vale da coleta de dados in loco, por pessoa não envolvida no trabalho ou no universo de análise ${ }^{(11)}$ e, também, de pesquisa documental em prontuários.

Como critério de inclusão, considerou-se que o período de internação da criança deveria ser de, no mínimo, 72 horas (três dias) na respectiva UTI-P. Já como exclusão, foi considerada a ausência de algum familiar/responsável dos pacientes pediátricos previamente à coleta de dados, o que não ocorreu. Desse modo, foram analisados 96 pacientes.

Na fase observacional, foram verificados os identificadores (nome da criança; nome do responsável; data de internação; data de nascimento; número do leito; e idade da criança) para identificação de todos os pacientes internados nas UTI-P, por meio do uso da placa de cabeceira do leito ou box e pulseira de identificação. Já a pesquisa documental foi realizada por meio da análise dos prontuários dos mesmos pacientes observados in loco previamente. Nesta etapa, foram verificados os itens de identificação dos pacientes nas últimas prescrições médicas e evoluções de enfermagem, a saber: nome completo; idade da criança; datas de nascimento e internação; número do leito; setor; diagnóstico/ número do prontuário; nome do responsável; e registro profissional.

Tanto para o registro das observações e pesquisa documental, foram utilizados formulários próprios elaborados para fins desta pesquisa, adaptados da Cartilha "10 Passos para a Segurança do Paciente", elaborada pela Rede Brasileira de Enfermagem e Segurança do Paciente. ${ }^{(12)}$ Tanto a coleta em observação sistemática não participante, como em fonte documental, culminou em extração manual das variáveis de identificação do paciente.

Todas as informações extraídas na observação e análise documental foram tabuladas em planilhas eletrônicas do software Microsoft Office Excel, versão 2010. Após isso, os dados dos três locais de estudo foram analisados em conjunto por meio de estatística descritiva, em medidas de proporção.

Todos os preceitos éticos que regem pesquisas com seres humanos dispostos na Resolução n.․ 466/2012 do Conselho Nacional de Saúde foram respeitados e o projeto que fomentou a presente investigação se encontra registrado sob parecer institucional n.. 866.802, de 2014.

\section{RESULTADOS}

Constatou-se que, dentre os 96 pacientes observados, 94 (98\%) eram identificados por meio de placa na cabeceira do leito ou porta do box e $2(2 \%)$ não apresentavam nenhuma forma de identificação. Apesar da identificação por placa na cabeceira do leito ou porta do box, a pulseira de identificação foi um recurso implantado e disponível nas três instituições estudadas, porém, não utilizado em nenhum paciente nas UTI-P. Na Tabela 1, constam informações da placa identificadora. 
Tabela 1 - Informações contidas na identificação do leito do paciente em Unidades de Terapia Intensiva Pediátrica. Sul do Brasil, 2015

\begin{tabular}{lccccc|}
\hline $\begin{array}{l}\text { Variável } \\
\text { (n=94) }\end{array}$ & $\mathbf{n}$ & Sim & \% & $\mathbf{n}$ & Não \\
\hline Nome da criança & 89 & 95 & 5 & 5 \\
Nome do responsável* & 73 & 78 & 21 & 22 \\
Data de internação & 89 & 95 & 5 & 5 \\
Data de nascimento & 51 & 54 & 43 & 46 \\
Número do leito & 91 & 97 & 3 & 3 \\
Idade da criança & 29 & 31 & 65 & 69 \\
\hline
\end{tabular}

*Inclui pai, mãe ou cuidador. Fonte: Dados da pesquisa

Dentre as identificações que constavam o nome da criança $(n=89), 62(70 \%)$ apresentavam o nome completo, nos demais $(n=27 ; 30 \%)$ constava apenas o primeiro nome.

Os resultados da análise realizada nos prontuários (prescrição médica e evolução de enfermagem) estão dispostos na Tabela 2.

Tabela 2- Identificadores contidos nos registros médicos e de enfermagem em Unidades de Terapia Intensiva Pediátrica. Sul do Brasil, 2015

\begin{tabular}{|c|c|c|c|c|}
\hline \multirow[t]{2}{*}{ Registros do prontuário } & \multicolumn{2}{|c|}{ Sim } & \multicolumn{2}{|c|}{ Não } \\
\hline & $\mathbf{n}$ & $\%$ & $\mathbf{n}$ & $\%$ \\
\hline \multicolumn{5}{|l|}{ Prescrição médica (n=96) } \\
\hline Nome completo & 89 & 93 & 7 & 7 \\
\hline Idade da criança & 93 & 97 & 3 & 3 \\
\hline Data de nascimento & 49 & 51 & 47 & 49 \\
\hline Data de internação & 62 & 65 & 34 & 35 \\
\hline Número do leito & 90 & 94 & 6 & 6 \\
\hline Setor & 96 & 100 & 0 & 0 \\
\hline Diagnóstico & 28 & 29 & 68 & 71 \\
\hline Número do prontuário & 63 & 66 & 33 & 34 \\
\hline Nome do responsável* & 12 & 12 & 84 & 88 \\
\hline Número de registro profissional & 51 & 53 & 45 & 47 \\
\hline \multicolumn{5}{|l|}{ Evolução de enfermagem (n=96) } \\
\hline Nome completo & 88 & 92 & 8 & 8 \\
\hline Idade da criança & 93 & 97 & 3 & 3 \\
\hline Data de nascimento & 57 & 59 & 39 & 41 \\
\hline Data de internação & 58 & 60 & 38 & 40 \\
\hline Número do Leito & 89 & 93 & 7 & 7 \\
\hline Setor & 40 & 42 & 56 & 58 \\
\hline Diagnóstico & 23 & 24 & 73 & 76 \\
\hline Número do prontuário & 51 & 53 & 45 & 47 \\
\hline Nome do responsável* & 31 & 32 & 65 & 68 \\
\hline Número do registro profissional & 24 & 25 & 72 & 75 \\
\hline
\end{tabular}

* Inclui pai, mãe ou cuidador. Fonte: Dados da pesquisa 


\section{DISCUSSÃO}

O presente estudo evidenciou que o recurso de identificação do paciente mais utilizado nas UTI-P dos hospitais estudados foi a placa identificadora, fixada na cabeceira do leito ou na porta do box $(\mathrm{n}=94$, $98 \%$ ). Outro aspecto que chamou a atenção foi o fato de nenhum paciente ter utilizado a pulseira de identificação.

O dado referido conflita com o estudo ${ }^{(8)}$ realizado em um hospital universitário do interior do Paraná, acerca da avaliação da identificação em pacientes adultos, o qual constatou que, de 1068 observações, $250(23,4 \%)$ não possuíam nenhum dos dois recursos de identificação do paciente (a placa de cabeceira do leito e a pulseira de identificação).

Apesar das UTI-P investigadas fazerem uso da placa identificadora, a falta de pulseira de identificação expõe as crianças a riscos de eventos adversos. Isso porque a ausência da pulseira pode dificultar a conferência da identidade do paciente, gerando extrema insegurança e necessita, portanto, de ações urgentes que atuem em prol da identificação e da segurança do paciente. ${ }^{(8)}$

O Protocolo de Identificação do Paciente, recomendado pelo Ministério da Saúde ${ }^{(5,7)}$ ressalta a importância da identificação do paciente no sentido de garantir o cuidado e assegurar que o tratamento seja realizado ao paciente a que se destina, evitando-se enganos e erros decorrentes do atendimento à saúde. Nesse contexto, o referido documento ${ }^{(7)}$ indica que a identificação deve ser realizada em todos os pacientes admitidos no ambiente hospitalar e em qualquer local que ofereça atendimento em saúde, tais como núcleos diagnósticos, laboratórios, etc., por meio de pulseiras de identificação, as quais são consideradas recursos seguros e capazes de reduzir possíveis incidentes e eventos adversos.

Reconhece-se que a utilização da pulseira de identificação se relaciona com o cuidado seguro. Esta afirmação é corroborada pelos resultados de um estudo realizado durante 45 meses e que identificou que mais da metade dos problemas com a identificação do paciente estavam relacionados com a falta de uso da pulseira de identificação. ${ }^{(13)}$

Com base no exposto, vislumbra-se uma perspectiva negativa dos achados deste estudo, porque a utilização da pulseira é recomendada como recurso nacional e internacional de identificação do paciente. Nesse sentido, o uso isolado de placas identificadoras na cabeceira do leito ou porta do box (98\%) pode inferir em riscos à saúde e segurança do paciente, maximizando o potencial de ocorrência de danos durante a internação em UTI-P.

Considera-se que a identificação do paciente pediátrico em regime de internação intensiva, por meio de placa na cabeceira do leito ou porta do box, apesar de ser uma demonstração de cuidado da equipe assistencial, não é suficiente para assegurar que o cuidado seja realizado para o paciente que se destina. Afinal, essa forma de identificação geralmente não o acompanha no momento da alta ou do transporte para realização de procedimentos diagnósticos ou terapêuticos fora da UTI-P, ou seja, são momentos que, somados à ausência da pulseira identificadora, podem afetar a segurança do paciente.

No caso de alta do paciente da UTI-P, pode-se ocorrer esquecimento da equipe quanto à troca da placa de identificação quando esta é fixada na porta do box, acarretando em oportunidade de erro de identificação na internação da próxima criança. Ademais, as placas de identificação à beira do leito não são transportadas junto ao paciente pediátrico para outros pontos de atenção à saúde e, portanto, reforça-se a necessidade de se (re)pensar o uso da pulseira identificadora.

Em UTI-P, são internados pacientes graves ou potencialmente graves, cuja idade variam de 29 dias de vida até 14 ou 18 anos, dependendo da rotina institucional. ${ }^{(14)}$ As crianças e adolescentes dessa faixa etária são acompanhados, normalmente, pelos pais ou algum responsável domiciliar que fornece as informações necessárias, tanto no sentido de confirmação da identificação do paciente internado, quanto a alguma particularidade referente ao doente. Porém, as características e condições clínicas dos pacientes, associadas ao grau de orientação dos pais ou responsáveis quanto às normas e rotinas setoriais, permitem o trânsito ou troca de leito das crianças internadas nas UTI-P, sendo outros possíveis fatores o risco de erro relacionado à identificação do paciente no caso da ausência da pulseira identificadora. 
Embora as UTI-P sejam ambientes controlados e tenham condições diferenciadas de trabalho em relação aos demais tipos de unidades de internação, a assistência prestada nesse serviço é altamente complexa, porque as rotinas de cuidado para a equipe assistencial são densas, a demanda de procedimentos intervencionistas diante de intercorrências é comum e a existência de diferentes condutas e doses terapêuticas de medicamentos para cada faixa etária são aspectos que tornam ainda mais perigosa a falta de identificação adequada do paciente. ${ }^{(15)}$ Com base nessa alusão, cumpre destacar o resultado que aponta a maior proporção de não identificação da idade do paciente nas placas identificadoras (principal recurso de identificação nas UTI-P), o que talvez possa favorecer um erro de dosagem em caso de medicações de emergência, por exemplo.

O uso de placas identificadoras próximo ao leito não exclui a necessidade de outros recursos de identificação, especialmente porque estas não contêm dados suficientes para assegurar a identificação correta do paciente, conforme evidenciam os resultados dessa pesquisa. Alguns autores afirmam que os eventos adversos podem continuar caso os pacientes não estejam usando a pulseira de identificação, se a mesma estiver com conformidade inadequada e, ainda, se seu uso não for racional. ${ }^{(16)}$

Uma pesquisa recente ${ }^{(17)}$ no contexto de UTI neonatal reforça o pressuposto anterior ao mencionar que a identificação dos pequenos pacientes por meio de pulseira, além do seu efetivo posicionamento, deve compreender também a checagem diária da presença e integridade da pulseira; a legibilidade dos identificadores; e a conferência sistemática da pulseira antes da prestação de cuidados, ponderações que coadunam ao próprio protocolo nacional de identificação do paciente. ${ }^{(7)}$

Apesar das placas identificadoras conterem o nome do paciente e a data de internação ( $n=89,94,6 \%)$, em $27(30,3 \%)$ constavam o primeiro nome da criança. Observou-se também que o principal identificador das placas foi o número do leito (Tabela 1). Este resultado atrela-se com a identificação das prescrições médicas e as evoluções de enfermagem, que também apresentavam falta de informações ou informações incompletas, inclusive falhas relacionadas ao registro do nome completo da criança (Tabela 2).

Uma interpretação importante de ser cumprida é o fato de que não identificar os pacientes por seu nome e priorizar aspectos institucionais, como o número do leito ou de teor biomédico como a causa de internação e/ou patologia, são aspectos que despersonalizam o cuidado e talvez favoreçam relações assistenciais menos humanizadas. A problematização expressa não se relaciona diretamente aos dados concretos, pois a maior proporção dos meios identificadores inclui o nome do paciente, ainda que não de forma completa. Porém, o nome do responsável foi deficiente nos registros em prontuários, tanto nas prescrições médicas como evoluções de enfermagem, e isso é sabidamente um aspecto importante quando se trata de assistência pediátrica.

Vale destacar que a alusão expressa configura-se como uma interpretação da realidade frente à identificação do paciente, o que não possibilita afirmar que, na dinâmica assistencial, a equipe de saúde trate as crianças por seu nome e conheçam a identificação de seus pais/cuidadores. Contudo, a notação destes identificadores nos registros médicos e de enfermagem possivelmente seria um aporte à segurança no cuidado, pois poderiam facilitar a recordação de qual paciente se registra determinada ação em prontuário.

Os dados obtidos referentes às placas identificadoras coadunam com as informações de um estudo realizado no ano de 2011, referente a eventos adversos relacionados à identificação do paciente, onde se constatou que entre 0 e $24,6 \%$ dos erros se referiam ao uso incompleto ou incorreto do nome do paciente. ${ }^{(13)}$ Este achado, sem dúvida, reforça o risco dos pacientes pediátricos internados nas unidades estudadas receberem cuidados inseguros.

Com relação ao prontuário do paciente (prescrição médica e evolução de enfermagem), a Joint Comission Resources ${ }^{(5)}$ indica que a identificação do paciente no prontuário deve conter no mínimo o nome completo do paciente, a data de nascimento, o nome completo da mãe e o registro profissional do executor do cuidado.

É válido enfatizar que o prontuário do paciente congrega os registros das informações referentes ao cuidado prestado pelos profissionais e por isso é fonte de dados para fins de auditoria e até de defesa, em casos de processos jurídicos e/ou éticos. ${ }^{(18)}$ Nessa ótica, alerta-se para que as instituições de saúde assumam a identificação do paciente como ponto crucial à segurança do paciente e dos trabalhadores e às próprias organizações. 
Ante o exposto, apesar da identificação via placa no leito/box aparentemente eficaz (ou ao menos, aderida), ainda existem espaços de melhoria, uma vez que há relação estrita entre identificação adequada, segurança do paciente e cuidado de qualidade. ${ }^{(19)}$ Outro aspecto a ser amplamente revisto é a utilização da pulseira de identificação que, apesar de disponível em todas as instituições estudadas, nenhuma criança fazia uso da mesma. Para minimizar o quadro exposto, sugerem-se ações como aquelas propostas pelo Ministério da Saúde ${ }^{(3,20)}$ que indica sensibilização e acompanhamento da equipe de saúde pelo Serviço de Educação Permanente aliada à atuação direta do Núcleo de Segurança do Paciente (NSP) das instituições de saúde.

O presente estudo abordou um tema de ampla relevância, a segurança do paciente. Frente à complexidade dos processos de trabalho nas instituições hospitalares, a identificação do paciente é de extrema importância e de responsabilidade multidisciplinar, uma vez que envolve a instituição, os profissionais, e a ativa participação dos usuários e familiares/acompanhantes. ${ }^{(7)}$

Considera-se como limitações deste estudo o fato de ter sido realizado apenas em UTI-P de hospitais públicos, o que não permite generalizações, além de os dados terem sido tratados apenas por estatística descritiva e de forma condensada entre as três instituições, o que fragiliza diagnósticos situacionais pontuais. Apesar disso, os resultados apresentados avançam no conhecimento e sustentam reflexões no âmbito do trabalho hospitalar que auxiliam na formação acadêmica, na pesquisa científica e no desenvolvimento profissional, especialmente por sinalizar a necessidade de se (re)planejar continuamente a identificação do paciente como meio de alavancar o cuidado seguro.

\section{CONCLUSÃO}

Constatou-se que as placas identificadoras na cabeceira do leito e porta do box são os recursos/ práticas de identificação do paciente mais utilizados, entretanto, em nenhum local os pacientes (crianças) usavam a pulseira de identificação. Outros aspectos relevantes das formas de identificação de pacientes em UTI-P como: ausência do uso de pulseira de identificação, dados incompletos na placa identificadora da cabeceira do leito e no prontuário do paciente.

Conclui-se que as práticas de identificação do paciente utilizadas comportam muitos identificadores, o que favorece a minimização de erros. No entanto, o uso isolado de placas identificadoras, a não utilização de pulseiras e a falta de informações no prontuário do paciente, confirmam a necessidade urgente de se fomentar barreiras de segurança nos serviços estudados.

À luz do cenário exposto, pondera-se que este estudo implica diretamente na busca atual por meios que incrementem a segurança do paciente. Em especial, a identificação do paciente é reafirmada como um aspecto primário e legítimo do cuidado seguro.

\section{REFERÊNCIAS}

1. Bohrer CD, Marques LGS, Vasconcelos RO, de Oliveira JLC, Nicola AL, Kawamoto AM. Comunicação e Cultura de Segurança do Paciente no Ambiente Hospitalar: Visão da Equipe Multiprofissional. Rev. EnfermUFSM. [Internet] 2016;6(1) [acesso em 10 ago 2016]. Disponivel:https://periodicos.ufsm.br/reufsm/article/view/19260/pdf_1.

2. World Health Organization (WHO). Quality of care: a process for making strategic choices in health systems. [Internet] Geneva: WHO; 2006 [acesso em 06 mai 2016]. Disponível:http://www.who.int/management/quality/assurance/ QualityCare_B.Def.pd.

3. Ministério da Saúde (BR). Relatório do seminário nacional para implantação do programa nacional de segurança do paciente. Brasília: Conselho Nacional de Saúde, Ministério da Saúde;2013. 11 p. 
4. Silva-batalhaEMS, Melleiro MM. Patient safety culture in a teaching hospital: differences in perception existing in the different scenarios of this institution. Texto contexto - enferm. [Internet] 2015;24(2)[acesso em 05 jun 2016.]. Disponível: http://dx.doi.org/10.1590/0104-07072015000192014.

5. World Health Organization(WHO). The Joint Comission International. Patient Identification.PatientSafety Solution. [Internet] USA: WHO/The Joint Comission International; 2007[acesso 10 jun 2016]. Disponível: http://www.who.int/ patientsafety/solutions/patientsafety/PS-Solution2.pdf.

6. Hoffmeister LV, de Moura GM. Uso de pulseiras de identificação em pacientes internados em um hospital universitário. Rev. Latino-Am. Enfermagem.[Internet] 2015;23(1) [acesso em 05ago de 2016]. Disponível: http://dx.doi. org/10.1590/0104-1169.0144.2522.

7. Ministério da Saúde (BR). Protocolo de Identificação do Paciente. Anexo 02. [Internet] Brasília: Ministério da Saúde; 2013 [acesso em 04 junho 2016]. Disponível: https://www20.anvisa.gov.br/segurancadopaciente/index.php/publicacoes/item/identificacao-do-paciente.

8. TresDP, Oliveira JLC, Vituri DW, Alves SR, Rigo DFH, Nicola AL. Qualidade da assistência e segurança do paciente: avaliação por indicadores. Cogitare enferm. [Internet] 2016;21(n.esp) [acesso em 07 nov 2016]. Disponível: http://dx. doi.org/10.5380/ce.v21i5.44938.

9. Porto TP, Rocha PK, Lessmann JC, de Souza S, Kretzer L, Anders JC. Identificação do paciente em unidade pediátrica: uma questão de segurança. Rev. Soc. Bras. Enferm. Ped. [Internet] 2011;11(2) [acesso em 02 mar 2016]. Disponível: https://sobep.org.br/revista/component/zine/article/142-identificao-do-paciente-em-unidade-peditrica-uma-questo-desegurana.html.

10. Mojica E, Izarzugaza E, Gonzalez M, Astobiza E, Benito J, Mintegi S. Elaboration of a risk map in a paediatric emergency department of a teaching hospital. Emerg Med J.[Internet] 2016;33(10) [acesso em 07set 2016]. Disponível: http:// dx.doi.org/10.1136/emermed-2015-205336.

11. Alvarez MEB. Organização, Sistemas e Métodos. 1 São Paulo: McGraw Hill; 1991.

12. Conselho Regional de Enfermagem do Estado de São Paulo (COREN-SP). Rede Brasileira de Enfermagem e Segurança do Paciente (REBRAENSP). Cartilha 10 passos para a segurança do paciente. [Internet] São Paulo: COREN-SP/ REBRAENSP; 2010 [acesso em 07 set 2016]. Disponível: http://portal.coren-sp.gov.br/sites/default/files/10_passos_seguranca_paciente_0.pdf.

13. Dhatt GS, Damir HA, Matarelli S, Sankaranarayanan K, James DM. Patient safety: patient identification wristband errors. ClinChemLab Med. [Internet] 2011;49(5)[acesso em 03 fev2016]. Disponível:http://dx.doi.org/10.1515/ CCLM.2011.129.

14. Ministério da Saúde (BR). Agência Nacional de Vigilância Sanitária. Resolução № 7, de 24 de fevereiro de 2010. Dispõe sobre os requisitos mínimos para funcionamento de Unidades de Terapia Intensiva e dá outras providências. Brasília: ANVISA; 2010.

15. Padilha KG, Vattimo MDFF, Silva SC, Kimura M. Enfermagem em UTI: cuidando do paciente crítico. São Paulo: Manole;2010.

16. Smith AF, Casey K, Wilson J, Fischbacher-Smith D. Wristbands as aids to reduce misidentification: an ethnographically guided task analysis. Int J Qual Health Care. [Internet] 2011;23(5) [acesso em 05 jan 2016]. Disponível: https:// doi.org/10.1093/intqhc/mzr045.

17. Gomes APTS, Querido DL, da Silva GRG, de Almeida LF, Rocha RG. The importance of newborn identification to delivery safe patient care. Cogitareenferm. [Internet] 2017;22(3) [acesso em 17 maio 2018]. Disponível: http://dx.doi. org/10.5380/ce.v22i3.49501.

18. Ministério da Saúde (BR). Sistema Nacional de Auditoria. [Internet] Brasília: Ministério da Saúde; 2011. [acesso em 03 de Agosto de 2016]. Disponível: http://www.sna.saude.gov.br/missao.cfm. 
19. dos Santos DS, Souza OV, Nascimento ANS, Pereira JS, Santos MJC, Alves MC, et al. Segurança do paciente: fatores causais de eventos adversos a medicamentos pela equipe de enfermagem.Ciências Biológicas e da Saúde. [Internet] 2014;2(2) [acesso em 02 mai 2016]. Disponível: https://periodicos.set.edu.br/index.php/cadernobiologicas/article/ view/1775.

20. Ministério da Saúde (BR). Fundação Oswaldo Cruz (FIOCRUZ). Agência Nacional de Vigilância Sanitária (ANVISA). Documento de referência para o Programa Nacional de Segurança do Paciente. [Internet] Brasília: Ministério da Saúde/ FIOCRUZ/ANVISA; 2014. 42p [acesso 13 setembro 2016]. Disponível: http://bvsms.saude.gov.br/bvs/publicacoes/documento_referencia_programa_nacional_seguranca.pdf. 